\title{
Global distribution of Trebouxiophyceae diversity explored by high-throughput sequencing and phylogenetic approaches
}

\author{
Sebastian Metz $\mathbb{C}^{1},{ }^{1}$ David Singer, ${ }^{2,3}$ \\ Isabelle Domaizon, ${ }^{4}$ Fernando Unrein ${ }^{1}$ and \\ Enrique Lara ${ }^{5^{*}}{ }^{\star}$ \\ ${ }^{1}$ Instituto Tecnológico de Chascomús (INTECH), \\ UNSAM-CONICET, Chascomús, Buenos Aires, \\ Argentina. \\ ${ }^{2}$ Laboratory of Soil Biodiversity, Institute of Biology, \\ University of Neuchâtel, Neuchâtel, Switzerland. \\ ${ }^{3}$ Department of Zoology, Institute of Biosciences, \\ University of São Paulo, Butantã, São Paulo, Brazil. \\ ${ }^{4}$ CARRTEL, INRA, Université Savoie Mont Blanc, \\ Thonon, France. \\ ${ }^{5}$ Real Jardín Botánico de Madrid, CSIC, Madrid, Spain.
}

\section{Summary}

Trebouxiophyceae are a ubiquitous class of Chlorophyta encountered in aquatic and terrestrial environments. Most taxa are photosynthetic, and many acts as photobionts in symbiotic relationships, while others are free-living. Trebouxiophyceae have also been widely investigated for their use for biotechnological applications. In this work, we aimed at obtaining a comprehensive image of their diversity by compiling the information of $\mathbf{4 3 5}$ freshwater, soil and marine environmental DNA samples surveyed with Illumina sequencing technology in order to search for the most relevant environments for bioprospecting. Freshwater and soil were most diverse and shared more than half of all operational taxonomic units (OTUs), however, their communities were significantly distinct. Oceans hosted the highest genetic novelty, and did not share any OTUs with the other environments; also, marine samples host more diversity in warm waters. Symbiotic genera usually found in lichens such as Trebouxia, Myrmecia and Symbiochloris were also abundantly detected in the ocean, suggesting either free-living lifestyles or unknown symbiotic relationships with marine

Received 12 February, 2019; revised 22 June, 2019; accepted 10 July, 2019. *For correspondence. E-mail enrique.lara@ rjb.csic.es; Tel. 349142030 17; Fax 34914200157. planktonic organisms. Altogether, our study opens the way to new prospection for trebouxiophycean strains, especially in understudied environments like the ocean.

\section{Introduction}

Trebouxiophyceae are a ubiquitous class of photosynthetic unicellular Chlorophyta (='green algae') encountered in many environments, including extreme ones such as hot (Büdel et al., 2009; Flechtner et al., 2013; Fučíková et al., 2014) and cold (Cavacini, 2001; Fermani et al., 2007; Hodač et al., 2016) deserts, or hyper acidic waters (Juárez et al., 2011). They are also common in mesophilic environments like soil and continental waters, some of them can also be found in the sea (Heesch et al., 2012; Tragin and Vaulot, 2018). But Trebouxiophyceae are mostly known for their symbiotic relationships with other organisms (Pröschold et al., 2011), where they act as photobionts, providing the by-products of their photosynthesis to the host. Most lichenizing fungi are indeed associated with Trebouxiophyceae (Thüs et al., 2011). Other mutualistic symbioses are also known, including diverse amoeboid protists (Gomaa et al., 2014; Lara and Gomaa, 2017), Metazoa (Aboal and Werner, 2011) and even vascular plants (Trémouillaux-Guiller and Huss, 2007). Some species lost secondarily their photosynthetic ability to evoIve as heterotrophic free-living organisms or parasites of diverse organisms (de Koning and Keeling, 2006; Pombert and Keeling, 2010), including humans (Lass-Flörl and Mayr, 2007). The variety of symbiotic associations including Trebouxiophyceae has motivated research for over 100 years (Pröschold et al., 2011).

Trebouxiophyceae have also attracted the interest of applied research. Indeed, green algae strains are generally simple to cultivate and allow the retrieval of a high biomass, which designated them for sustainable biogas production (Huss et al., 1999; Safi et al., 2014). Other strains also produce secondary metabolites of medical interest, for instance anticancer drugs (Safi et al., 2014). These individual examples reflect the economic and biotechnological importance of Trebouxiophyceae and 
justifies the research effort in the prospection for new strains with potentially promising properties. In 2012, the online reference site for algae (http://www.algaebase.org) documented 546 described Trebouxiophyceae species (Guiry and Guiry, 2018). This number has been raised 3 years later (October 2018) to 857 (Guiry and Guiry, 2018), certainly fostered by the generalization of genetic barcoding to discriminate between morphologically similar strains.

This prospection is, however, not straightforward. Indeed, identifying strains based on their morphology is often impossible, and while certain Trebouxiophyceae present conspicuous morphologies (Lewis and McCourt, 2004), the bulk of their diversity cannot be readily distinguished from each other (i.e. 'green balls', most of all formerly classified into a highly paraphyletic genus 'Chlorella') despite being genetically and physiologically very heterogeneous. The lack of flagellar apparatus reduces even more the number of possible morphological discrimination criteria to apply, which resulted in an underestimation of their diversity (Krienitz et al., 2015). The advent of molecular methods permitted establishing a taxonomic framework, resulting in splitting the vast genus Chlorella into many monophyletic genera, providing a framework for the classification of strains of evolutionary, ecological or biotechnological interest (e.g. Bock et al., 2011; Krienitz et al., 2015; Neofotis et al., 2016).

Environmental DNA approaches based on high throughput sequencing of amplicons of a chosen marker gene are nowadays the silver bullet technique for prospecting microbial diversity. This type of approach can be applied to detect peaks in diversity and evaluate the genetic divergence of the environmental sequences as compared to reference databases ('genetic novelty'; Mahé et al., 2017). It provides therefore the needed information on where to look for undescribed organisms. In the case of the Trebouxiophyceae, given the size of the reference database and the fact that many deposited strains have been barcoded, it can be safely assumed that most novel sequences correspond to potentially undescribed organisms.

Here, we applied an environmental DNA approach based on the V9 variable region of the gene coding for the SSU rRNA to a wide sampling design including samples taken from freshwater (FW) and soil (SO) samples, plus all sequences obtained in the course of the TARA Oceans expedition (de Vargas et al., 2015), for a total of 435 samples spanning a vast range of climates, and environmental parameters. Trebouxiophyceae-related operational taxonomic units (OTUs) were placed in a reference phylogenetic tree based on both cultured organisms and environmental sequences and assigned to the existing genera using evolutionary placement algorithm (EPA) (Zhang et al., 2013). Our aim was to obtain an overview of the distribution of trebouxiophycean diversity, infer which environments would host the largest unknown diversity, and also the most genetically diverse clades. Also, we aimed at detecting the presence of symbiotic clades in environments where they have not been reported, suggesting unreported biotic associations. Because of the importance of the salinity barrier (Logares et al., 2009), and also due to the stress associated with desiccation, we hypothesize that freshwater, soil and marine communities will differ in their composition. We also expect finding more genetic novelty in marine environments, as knowledge on oceanic Trebouxiophyceae is nowadays still very limited. In line with better-known soil and freshwater systems, we expect that marine environments should also host symbiotic associations; this would be suggested by the frequent presence of known symbiotic genera such as Symbiochloris for instance.

\section{Results}

\section{Phylogenetic tree construction and annotation}

In total, we extracted 2018 sequences from the public databases SILVA and NCBI that belonged unambiguously to Trebouxiophyceae (Table S1). Amongst them, we used 1814 high quality (no errors or ambiguous nucleotides), long (i.e. $>1000 \mathrm{nt}$ ) and non-redundant sequences to build the reference phylogenetic tree. Most of the main Trebouxiophyceae clades were supported with high bootstrap values $(>70 \%)$ and annotated at the genus level. Those that have bootstrap values lower than $70 \%$ were annotated at the order level according to the bibliography (Leliaert et al., 2012; Lemieux et al., 2014). Three formerly uncharacterized deep clades were retrieved and annotated as TREB01-TREB03 (Fig. 1). These clades were composed exclusively by environmental sequences that had been already published previously.

The reference phylogenetic tree was used to classify 961 OTUs from our environmental DNA survey. We identified 11 new, deep branching, clades. Amongst them, nine could be potentially assigned to new genera as compared with the average genetic distances that separate trebouxiophycean lineages in the phylogenetic tree (Fig. 1), and the two remaining others branched that are at the deepest nodes could be assigned to a suprageneric status. Three lineages were marine and the remaining eight were shared between SO and FW.

\section{General diversity patterns}

The final data set included 63 samples from FW, 33 from SO and 107 samples from MA (Fig. 2, Table S2 for FW and SO, Supplementary Material from de Vargas et al., 2015 for MA). The distribution of Trebouxiophyceae across samples and environments were not homogeneous. Their contribution to the totality of sequences per sample was, in average, $1 \%$ in FW samples, but ranged between $0.01 \%$ and $24 \%$; 


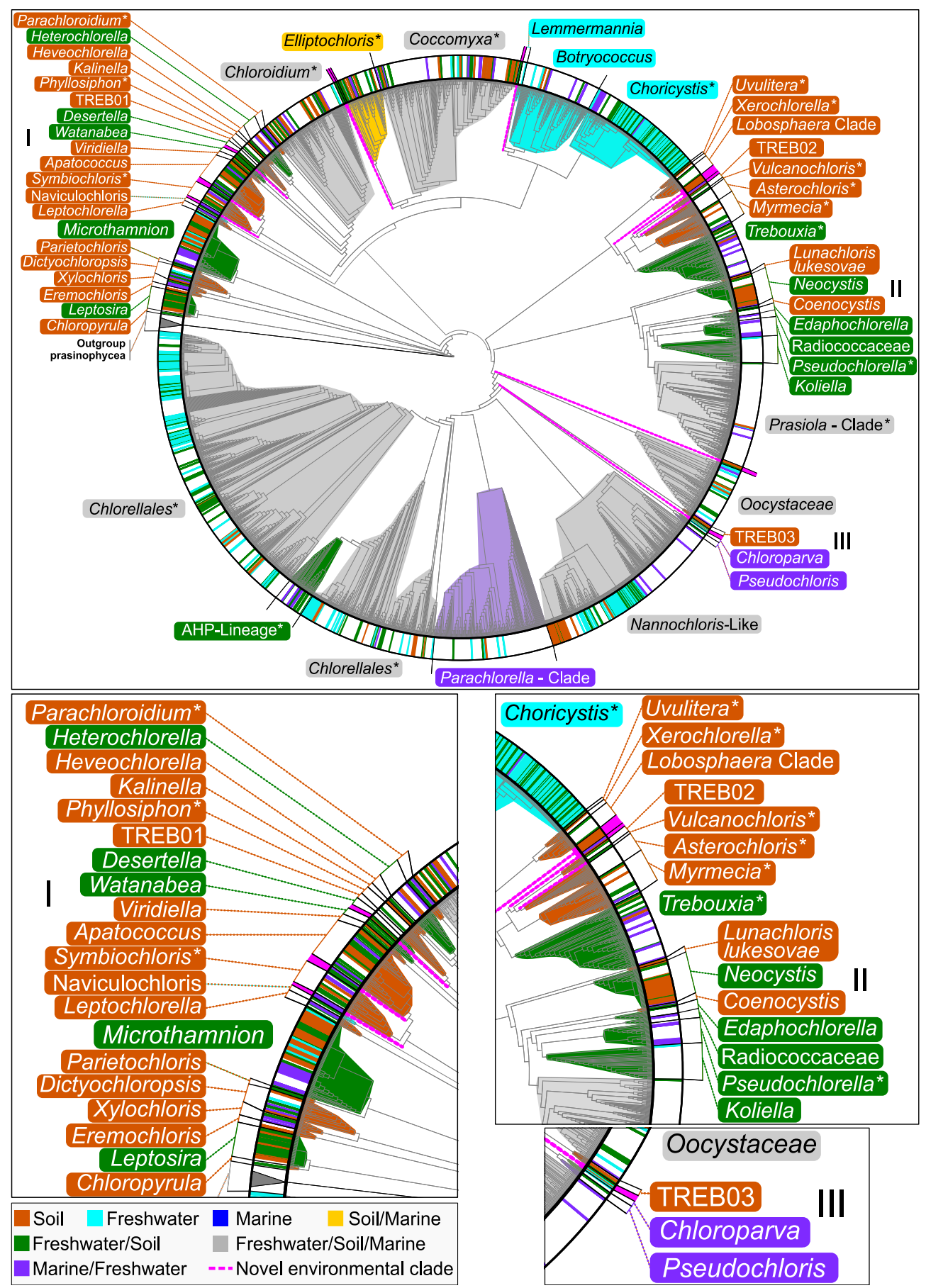

Fig. 1. Reference tree with sequences annotations. Colours of the clade names illustrate the environments types (SO, FW and MA) where the corresponding organisms have been found before this study. Inner circle: reference tree; colours indicate environment types from where the reference sequences (isolates and environmental clones) were found. The asterisks correspond to clades that contain symbiotic organisms. Branches in purple correspond to novel deep branching lineages discovered in this study. Outer circle: lines correspond to OTUs found in this study. Colours indicate the environment type from which they originate. Numbers I, II and III correspond to regions of the tree that have been illustrated in detail, notably illustrating the OTUs from symbiotic groups that have been found in MA (genera Trebouxia, Myrmecia, Pseudochlorella and Symbiochloris). [Color figure can be viewed at wileyonlinelibrary.com]

$0.44 \%$ in SO samples, with a minimum and maximum contribution between $0.006 \%$ and $2.95 \%$; and $0.16 \%$ in MA samples ranging between $0.01 \%$ and $3 \%$. Within these samples, a total of 200,747 sequences were affiliated to Trebouxiophyceae. FW contributed with $80.73 \%$ of the total sequences, SO and MA with $15.79 \%$ and $3.48 \%$ 


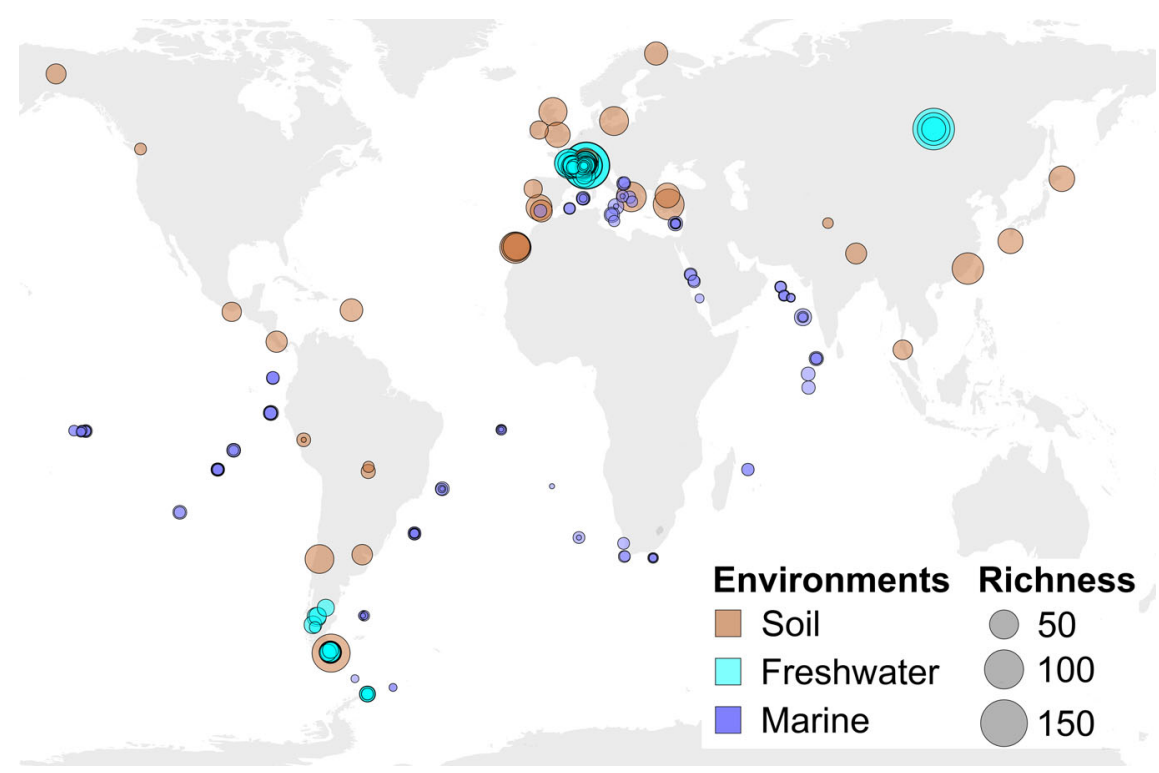

Fig. 2. Map including all sites in the study. Numbers of Trebouxiophyceae OTUs sequences found on each sample are indicated by the diameter of the circles. Colours indicate the environment types from where the sample has been taken. [Color figure can be viewed at wileyonlinelibrary.com] respectively. All sequences clustered into 961 OTUs (Tables S3 and S4). From these, 298 were found in FW, 227 in SO and 90 in MA, while 346 OTUs were found in both FW and SO. MA did not share any single OTU with any other environments. The non-metric multidimensional scaling (NMDS) analysis showed a clear separation between SO and FW (Fig. 3). Further, irrigated and mostly wet soils were placed in an intermediate position between FW and SO experimenting drought (Fig. 3). Two wet/irrigated soils respectively from a bog in Pto. Williams in extreme Southern Chile (SO_10) and an agricultural soil from the Danish Island Bornholm (SO_90) fell between soils experimenting drought. A careful inspection of their composition showed that an overrepresentation of common OTUs from dry soil, Leptosira (OTU_89645) and Neocystis (OTU_89623) overrepresented in SO_10; and Chloroidium (OTU_89851) in SO_90 (Table S4).

FW was dominated by Chlorellales $(48.7 \%$ of sequences), Choricystis (31\% of sequences), TREB03HTS (14.3\% of sequences) and Nanochloris-like sequences ( $1.8 \%$ of sequences) (Table S5). Most of these groups were highly diverse. Chlorellales contributed with 208 OTUs, Choricystis with 138 and Nannochloris-like with 77 OTUs. The most widespread clade was TREB03HTS, a previously undetected clade present in $58 \%$ of all FW samples, and represented by four OTUs in total. The most widespread OTU belonged to symbiotic genus Coccomyxa (OTU_89751) and was present in $77.7 \%$ of all samples, though its average contribution was low $(0.4 \%$ of total FW Trebouxiophyceae sequences). The most abundant OTU was the Chlorellales affiliated OTU_14045 that contributed with $42 \%$ of the total FW Trebouxiophyceae sequences and was present in $66.7 \%$ of all FW samples (Table S5).
SO was dominated by Chlorellales (18\% of all SO sequences; 90 OTUs), genus Apatococcus (12\% of sequences; 27 OTUs) and genus Chloroidium (11\% of sequences; 33 OTUs). Interestingly, the most widespread OTU in SO was OTU_89751 (genus Coccomyxa), the same as in FW, and was present in $87.9 \%$ of total SO samples, also in low abundance ( $6.4 \%$ of all SO sequences).

MA was dominated by genera Chloroidium (37.4\%, 4 OTUs) and Apatoccocus (8.3\%, 2 OTUs) respectively. The most frequent OTUs belonged to Heterochlorella

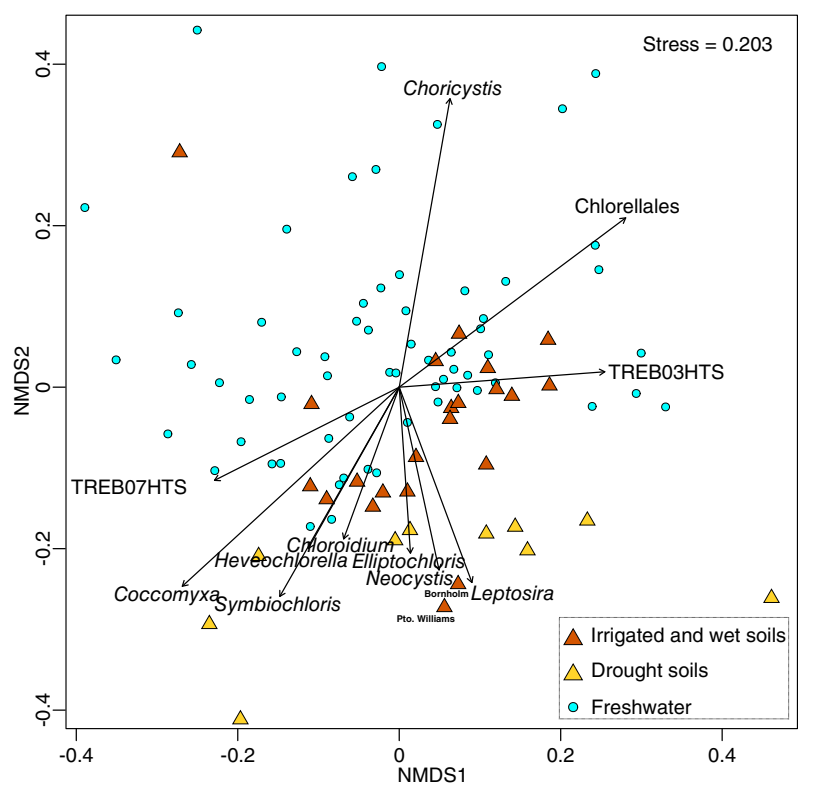

Fig. 3. NMDS indicating the relationships between soil and freshwater communities. [Color figure can be viewed at wileyonlinelibrary.com] 
(43.9\% of the samples), newly detected environmental clade TREB10HTS (40.2\% of the samples), Coccomyxa and Chloroidium (30.8\% and $24.3 \%$ of the samples respectively). Marine Trebouxiophyceae peaked in diversity in the Mediterranean and were distributed almost evenly between surface waters $(53 \%)$ and deep chlorophyll maximum (47\%). Most of the OTUs from FW and MA (638 OTUs of 734) derive from the pico sized fraction, in the samples where plankton was sequentially filtered $(0.8-5 \mu \mathrm{m})$.

Some OTUs were observed exclusively in cold environments in several distant locations, suggesting adaptations to cold temperatures (Fig. 2, Table S4). For instance, OTU_109033 (genus Coccomyxa) was well represented in Antarctic samples and was also present in other cold environments such as Argentinean Patagonia and Alaska, both in SO and FW. Other clades containing OTUs possibly exclusive to cold environments were TREB06HTS and TREB07HTS. On the other hand, OTUs affiliated with TREB04HTS (OTU_89644 and OTU_89739) and TREB08HTS (OTU_117567) were found exclusively but consistently in all samples from Lanzarote (Canary Islands), i.e. under desertic climate. Some clades containing many (or mostly) symbiotic forms usually found in lichens were also found in MA samples. These clades correspond to genera Apatococcus, Heterochlorella, Myrmecia, Symbiochloris, Trebouxia, family Radioccocaceae and the order Prasiolales.

\section{Genetic novelty}

About $80 \%$ of all OTUs shared more than $95 \%$ identity with published sequences in the $\mathrm{PR}^{2}$ eukaryotic ribosomal database (Guillou et al., 2013). However, present knowledge on Trebouxiophyceae genetic diversity was not evenly

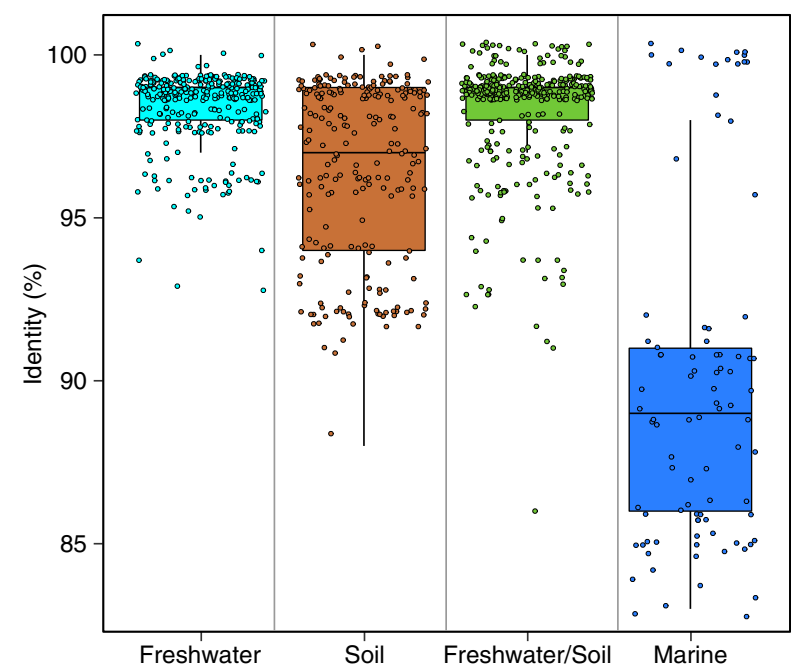

Fig. 4. An illustration of genetics novelty of OTUs in all three environment types, showing the percentage of identity for each OTU as aligned with all $\mathrm{PR}^{2}$ entries. [Color figure can be viewed at wileyonlinelibrary.com] distributed. Only $20 \%$ of MA OTUs had more than $95 \%$ of identity with $\mathrm{PR}^{2}$ reference sequences. In contrast, this percentage rose above $89 \%$ in FW and SO (Fig. 4, Table S3).

\section{Discussion}

A reference phylogenetic tree to classify Trebouxiophyceae environmental molecular diversity

Our reference phylogenetic tree is a compilation of all knowledge on Trebouxiophyceae diversity available on public databases; indeed, all genera described to date have at least one representative species barcoded for the 18S SSU rRNA gene (Leliaert et al., 2012; Lemieux et al., 2014). Leliaert et al. (2012) predicted the existence of seven major groups amongst Trebouxiophyceae. Even if the limitation of the SSU rRNA gene for phylogenetic Trebouxiophyceae studies has been previously reported (Neustupa et al., 2013; Fučíková et al., 2014), our analysis retrieved the seven predicted groups as well-supported clades most often with high bootstrap values $(>70 \%)$. Moreover, our study reveal three uncharacterized clades from soil, evidencing the existence of an unsuspected deep diversity (i.e. arguably at the genus level or above). Our reference phylogenetic tree can be therefore considered as a useful tool to approach new ecological and evolutionary questions (Pan et al., 2017) and classify the unknown sequences (Mahé et al., 2017) like it has already been done for other groups of eukaryotic microorganisms (del Campo et al., 2018).

\section{Environmental Trebouxiophyceae diversity and promising environments for new strain prospection}

Despite our large sampling effort our rarefaction curves reached saturation only in MA (Fig. S1). This indicates that we did not cover all diversity in FW and SO, and that environmental prospection may still reveal more undetected lineages. Still, the number of OTUs estimated for the whole study (961) was higher than the number of species estimated for class Trebouxiophyceae (857 species; Guiry and Guiry, 2018), knowing that the V9 region of the 18S rRNA gene from many published sequences are partial and not are resolutive enough to discriminate some organisms at the species level (Tragin et al., 2018).

Altogether, our findings show that Trebouxiophyceae diversity is highest in FW habitats followed by the SO habitat. This contrasts with MA, where only a limited number of OTUs have been detected despite a higher number of samples surveyed. The ocean is, however, of high interest for prospecting new forms, as genetic novelty in marine systems was highest, suggesting a high potential for new strains with novel properties. Indeed, only $20 \%$ of all sequences shared $95 \%$ or more similarity with sequences deposited in $\mathrm{PR}^{2}$. In MA samples, subtropical and tropical oceans (Mediterranean, Red 
Sea, central Indian Ocean) seemed to host a larger diversity than colder regions (Fig. 2) suggesting a latitudinal gradient of diversity as observed in marine bacteria (Fuhrman et al., 2008). Warm oceans are therefore most relevant environments for prospection for new Trebouxiophyceae strains. Filtering seems to be an adequate strategy to enrich environments in Trebouxiophyceae. Indeed, most (40.5\%) sequences from MA and $(98.7 \%)$ from $\mathrm{FW}$, data sets have been retrieved from 'pico' fraction $(0.8-5 \mu \mathrm{m})$ suggesting that a large part of the Trebouxiophyceae diversity is composed of tiny-sized organisms. This assumption is confirmed by several works based on flow cytometry that found Trebouxiophyceae to dominate picoeukaryotic diversity in certain freshwater assemblages (Pálffy et al., 2014; Metz et al., 2019).

The marked difference between MA and FW/SO floras confirms our hypothesis, and follows well-known patterns as the salinity barrier represent a major physiological divide for prokaryotic and eukaryotic microorganisms alike (Logares et al., 2009). The transition across the salinity barrier has been apparently achieved in the freeliving genus Microthamnion where our analysis revealed a large, deep branching clade sister to all other species exclusively composed of marine sequences (Fig. 1). Further investigations directed towards the isolation of these organisms may lead to the splitting of Microthamnion, given the respective position of MA and FW sequences and reveal organisms with emerging properties. Other supposedly exclusive soil clades have been found in the sea, such as the exclusively free-living Xylochloris, Dichtyochloropsis, and family Radiococcaceae (Fig. 1). Genus Picochlorum (included within Nannochloris - Like; Table S1) well-known for its tolerance towards large variations in salinity has been isolated accordingly from marine and brackish environments alike (Foflonker et al., 2018). However, in our study, these organisms appeared only in MA samples, possibly; even if they tolerate freshwater, it is still unclear if they can form viable populations under low salinity conditions.

$\mathrm{SO}$ and FW are more similar to each other in their taxonomic composition, yet our NMDS analysis (Fig. 3) illustrates a trend for a separation between SO and FW. SO can be differentiated further in two main categories, 'wet' soils (agricultural soils that are irrigated, moist forest soils and peat), that are generally situated between FW and the second main category, 'dry' soils that experiment drought (most natural soils) as can be interpreted in Fig. 3. The differences between these communities shows that drought is also an important driver for Trebouxiophyceae diversity. Moreover, this suggests that intermediate environments like wet soils may have been used as an evolutionary stepping-stone towards transition between FW and dry soil. This evolutionary scenario has been proposed as a possible way for colonizing emerged lands by the ancestors of modern plants (de Vries and Archibald, 2018).

Typical 'dry' SO genera were the free-living Leptosira and Neocystis, and also the lichen symbionts Symbiochloris and Coccomyxa, while FW genera were Choricystis and Chlorellales (Fig. 3). There are, however, single OTUs that seem to transcend these barriers like OTU_13937 (uncharacterized clade TREB03HTS) and OTU_14045 (Chlorellales), both abundant in FW and SO, including 'dry' soils (Table S4). In contrast to MA samples, $89 \%$ of all FW and SO sequences have $95 \%$ or more similarity with sequences published in $\mathrm{PR}^{2}$ (Fig. 4), which illustrates a better knowledge of the trebouxiophycean flora of these environments. Still, the most common and abundant OTUs in FW and SO environments correspond to undescribed organisms (OTU_14045, OTU_13937), which illustrates the fact that both SO and FW host also great potential for discovery.

In total, we found eight deep-branching, genus level clades. Two of these clades, namely TREB05HTS and TREB06HTS, were composed with OTUs that were exclusively found in cold environments such as Antarctica, Tierra del Fuego, Patagonia and, in the Northern Hemisphere, Northern Finland/Lappland (Table S4). In contrast to what has been found in MA, cold environments did not host significantly lower diversity than warmer environments in SO and $\mathrm{FW}$, and the richest FW samples were alpine Lake Brévent (287 OTUs), a high altitude lake $(2100 \mathrm{~m})$ covered with ice 8 months per year, and Lake Baikal (198 OTUs); the richest soil sample was originated from subantarctic Puerto Williams (147 OTUs). A systematic prospection of cold environments may reveal new psychrophilic strains with potentially genomic adaptations to cold environments, like the polar Trebouxiophyceae Coccomyxa subellipsoidea (Blanc et al., 2012).

Desert environments, characterized by low humidity and extreme UV radiation levels may host also organisms of potential interest for biotechnology. Two OTUs were observed only in samples retrieved from these environments, OTU_89739 (affiliated to uncharacterized clade TREB04HTS) and OTU_89758 (Botryoccocus $\mathrm{sp}$.); interestingly, this genus includes several strains that are well-known for their biotechnological application in the industry of biofuel production (Metzger et al., 1991; Metzger et al., 1990; Metzger and Largeau, 1999; Banerjee et al., 2002; Raja et al., 2008). A wider prospection of these regions would be most wanted to characterize their diversity and estimate their potential for novel diversity discovery.

\section{Novel possible symbiotic associations}

Several trebouxiophyceaen genera are well-known to have the tendency of building symbiotic relationships with 
lichenizing fungi. These associations are generally found in subaerial environments like rocks, trunks and soil, but not in permanently wet environments (Hawksworth, 2000). Therefore, finding these organisms in aquatic environments, and especially the ocean, suggests symbiotic associations that have not been reported previously. Recently, the ability to live as a symbiont was proposed as a mechanism to adapt to saline environments (Hinojosa-Vidal et al., 2018). Although many marine protists are known to host symbiotic photosynthetic eukaryotes, there are little mentions of Trebouxiophyceae involved in such relationships. A probable case is the association between a 'Chlorella' (to be considered as a Trebouxiophyceae sensu lato) and a foraminiferan (Lee et al., 1982), or between Elliptochloris marina and the sea anemone Anthopleura (Letsch et al., 2009). However, it is not unlikely that other similar associations remained overlooked given of the lack of characteristic morphological features of most Trebouxiophyceae.

Genera Myrmecia, Symbiochloris and Trebouxia are species-rich genera that include a majority of lichen photosymbionts (Honegger and Brunner, 1981; Rambold et al., 1998; Škaloud et al., 2016). Other genera, such as Apatococcus, Coccomyxa, Elliptochloris and Pseudochlorella comprise a variety of lichen symbionts and free-living forms. In this study, we detected 33 OTUs belonging to these clades present in marine systems. Some can be considered as abundant, representing over $1 \%$ of the whole community in certain MA samples (Table S4). We consider unlikely that these sequences correspond to organisms of edaphic origin brought in the sea as we systematically removed rare sequences. Moreover, the marine sampling was performed far away from the coast (de Vargas et al., 2015). Some of these organisms may possibly be free-living forms from these groups or, perhaps, free-living stages of symbiotic organisms. In support for this hypothesis, two OTUs (OTU_59ee09bd8eccf066d00d 755548d60655 and OTU_a0cbafb71d700506213b5e4b3c3 870dc) were classified within Elliptochloris; a future expansion of the trebouxiophyceaen genetic database will help determine how far these organisms are related to Elliptochloris marina or if they constitute a new symbiotic lineage. Althogether, these findings open the way for an accurate assessment of Trebouxiophycean symbiotic associations in marine systems, their range of possible hosts and the relevance of these associations at ecosystem level.

\section{Concluding remarks}

Environmental DNA surveys based on large sampling designs are excellent strategies to investigate diversity hotspots, especially for groups with homogeneous morphologies such as Trebouxiophyceae. Here, we identified the environments where the bulk of global diversity is to be found (freshwater, soil), but also where the potential for novel discovery is the highest (marine plankton), and where potentially unknown symbiotic associations take place. This sets a starting point for organism centered research, based on cultures, with potential implications ranging from evolutionary studies focused on symbiosis to the retrieval of new strains with relevant biotechnological properties.

\section{Experimental procedures}

Curated database and the reference phylogenetic tree reconstruction

The reference phylogenetic tree was built with a similar pipeline as proposed by del Campo et al. (2018). Briefly, sequences related to Trebouxiophyceae were extracted from SILVA v132 database (Quast et al., 2012). They were aligned with MAFFT v7.123 (Katoh and Standley, 2013). Introns and intergenic regions were excluded using Geneious v9.0.5 (Biomatters, Auckland, New Zealand). Sequences were clustered at $99 \%$ of identity with USEARCH v10.0.24 (Edgar, 2010). The centroids for each cluster were aligned with MAFFT and a phylogenetic tree inference with RaxML v8.2.8 (Stamatakis, 2014) using GTR + GAMMA model and 100 bootstraps, after the noninformative regions of the alignment excluded by trimAL v1.4.rev22 (Capella-Gutierrez et al., 2009) was performed. The phylogenetic tree was manually curated and these steps were iteratively repeated until obtaining a consistent database with sequences that represent most of the clades (Leliaert et al., 2012).

Sequences belonging to Trebouxiophyceae but not assigned as such in GenBank (like unassigned environmental clones) were searched into the NCBI database (September 2017). The search was performed with BLAST v 2.6.0 (Altschul et al., 1997). For each sequence, 100 hits with more than $97 \%$ sequence identity, e-value less than $1 \mathrm{E}-6$ and bit-score higher than 1000 were retrieved. This step was repeated until no new sequences were obtained. Sequences with $18 \mathrm{~S}$ mark region length shorter than 500 nucleotides were excluded using the filtration step proposed by Logares (2017). New sequences were manually revised using web BLAST with standard parameters in order to exclude non-Trebouxiophyceae sequences.

To build a reference phylogenetic tree, 1814 sequences with more than 1000 nucleotides and not redundant were used to made an initial alignment with MAFFT using the EINS-I algorithm (Katoh and Standley, 2013), which we automatically trimmed poorly aligned regions with trimAL (Capella-Gutierrez et al., 2009). Sequences selected from each clade were aligned with MAFFT (Katoh and Standley, 2013) using E-INS-I algorithm and 1000 replicates, the alignment was edited and a phylogenetic tree was performed using RaxML (Stamatakis, 2014) with GTR + GAMMA model and 1000 bootstraps. This tree was used as a reference to 
placement environmental sequences from high throughput sequencing into the different Trebouxiophycean clades or genera.

\section{Phylogenetic tree annotation}

The names of clades from the reference tree were assigned according to the bibliography (Leliaert et al., 2012; Fučíková et al., 2014; Hodač et al., 2016). We defined new environmental clades (designed as TREBXX) when environmental sequenced clustered together in a robust clade supported by a bootstrap value of at least $70 \%$. We annotated original environment type (FW, MA or SO) and lifestyle (photosymbionts/parasites) based on information retrieved from Genbank and on the bibliography. Organisms found in brackish environments were classified as marine as the salinity barrier is considered as the greatest divide in microbial diversity (Logares et al., 2009). This information was deposited in Table S1. OTUs were added to the Trebouxiophyceae reference alignment using PaPaRa v2.5 (Berger and Stamatakis, 2011) and placed in the reference phylogenetic tree with EPA implemented in RaxML v 8.2 and - epa-accumulated-threshold $=0.999$ command (Zhang et al., 2013; Mahé et al., 2017). The best placements for each OTU were selected using Gappa (https://github.com/ Iczech/gappa) and corroborated using BLASTN web service tool (Altschul et al., 1997). New clades obtained in this study were annotated as TREBXXHTS. The phylogenetic tree figure was performed using Itool (Letunic and Bork, 2007) and edited with inkscape 0.48 .4 (https://inkscape.org).

\section{Sampling, DNA extraction, amplification and sequencing}

SO samples $(0.5 \mathrm{~g})$ were stored in a buffer appropriate for environmental nucleic acids preservation (Life Guard, Qiagen) prior to DNA extraction (see Seppey et al., 2017). FW samples consisted in plankton that were pre-filtered at different size fractions, with an initial pre-filtration through $50 \mu \mathrm{m}$ mesh (Table S2). The filters were placed in cryovials with $1.8 \mathrm{ml}$ of lysis buffer (40 mM EDTA, $50 \mathrm{mM}$ Tris- $\mathrm{HCl}$, $0.75 \mathrm{M}$ sucrose) and stored at $-80^{\circ} \mathrm{C}$ until DNA extraction (see Schiaffino et al., 2016). Nucleic acids extraction was conducted using the kit PowerSoil ${ }^{\circledR}$ DNA Isolation Kit (Qiagen). PCR amplification of the v9 region of the gene coding for the SSU rRNA gene was performed using the primers $1389 \mathrm{~F} 5^{\prime}$ - TTGTACACACCGCCC $-3^{\prime}$ and $1510 \mathrm{R}$ 5'- CCTTCYGCAGGTTCACCTAC (Amaral-Zettler et al., 2009), and the same protocols as in de Vargas et al. (2015). For MA, the different size and depth fraction as described in de Vargas et al. (2015) were analysed individually.

\section{Bioinformatic analysis}

Our initial data set was constituted of 435 samples distributed in different three different environments: 36 Soil (SO),
65 Freshwater (FW) and 334 Marine (MA). For details about the geographical distribution and the reference of each sample see Table S2 for SO and FW, and supplementary material from de Vargas et al. (2015) for MA; all these correspond to open ocean samples. The data set and the sequences of de Vargas et al. (2015) are available in http://taraoceans.sb-roscoff.fr/EukDiv/. In order to compare the three environments, we processed the FW and the SO samples bioinformatically (i.e. assembling, curation, parsing) with the same pipeline developed by de Vargas et al. (2015) and already applied in (Singer et al., 2016). Likewise, Tara Ocean and our own samples were clustered with Swarm v2.2.2 (Mahé et al., 2014) with the recommended parameters. The swarm seeds (OTUs) were assigned using $\mathrm{PR}^{2}$ v 4.10.0 (Guillou et al., 2013) and sequences affiliated with Trebouxiophyceae were extracted. New sequences were deposited in NCBI under the numbers MK988625-MK989495.

\section{Generation of the community matrix (OTU table)}

In order to estimate abundance for each OTU, we first drew a high-throughput environmental sequences (HTES) table, defined as a contribution of all dereplicated sequences to each sample. Finally, for each OTU we searched all HTES that compose them. Because we wanted to consider only samples that contained a significant population of Trebouxiophyceae, samples with less than 10 sequences in total were excluded. Indeed, the ecological relevance of rare sequences in lllumina-based environmental diversity survey can be subject to controversy (Schiaffino et al., 2016). All obtained OTUs with their respective affiliations are detailed in Table S3.

The rarefaction curve was performed by each sample, each environment (Freshwater, Soil and Marine) and for all OTUs together using the VEGAN version 2.5-3 package (Oksanen et al., 2008) and R (http://cran.r-project.org).

\section{Ordination and global distribution of Trebouxiophyceae and genetic novelty}

To highlight the heterogeneity between the environments, we performed a non-metric multidimensional scaling (NMDS). We used only the samples from SO and FW and exclude those from MA as they did not share any OTU with the others environments. Moreover, SO samples were divided into irrigated and mostly wet soils (like forest soil and peat) and others soils experimenting drought, to be displayed differentially in the figure. We normalized our data set with a Hellinger transformation (Legendre and Gallagher, 2001) and used a Bray-Curtis distance to compute the NMDS using VEGAN 2.5-3 package (Oksanen et al., 2008) package from R 3.5 (http://cran.r-project.org). Different biotic variables, calculated as a contribution for 
each clade to each sample were fit in the NMDS with the function envfit from VEGAN 2.5-3, only variables with significant $p$-value ( $p$-value $<0.01)$ were shown in Fig. 3.

To assess the global distribution of Trebouxiophyceae, we calculated the specific richness of each samples, here defined as the total number of OTUs. We plotted the three environment independently on a world map, to see in which environment the samples were more diverse. We used GGMAP version 2.6.1 and GGPLOT version 3.1.0 package from $\mathrm{R}$ to compute the analysis. To determine the representation of the OTUs in references databases and in each environment, OTUs were separated according to their distribution, forming four classifications (Freshwater, Soil, Freshwater and Soil, and Marine). For each OTU we extracted the percentage of identity assigned in the classification step explained before using $\mathrm{PR}^{2}$ (Guillou et al., 2013) reference database and plotted in boxplot graphs using a GGPLOT version 3.1.0 from $R$ (http://cran.r-project.org).

\section{Conflict of interest}

The authors declare that there is no conflict of interest regarding the publication of this article.

\section{Acknowledgements}

We are indebted to Didier Debroas for providing several freshwater samples from French lakes. EL wishes to acknowledge the program 'Atracción de talentos de la Comunidad de Madrid' (grant 2017-T1/AMB-5210 4) and Swiss National Fund project SNF 31003A_163254 for funding. The project was funding by the ANPCyT 'Agencia Nacional de Promoción Científica y Tecnológica' (PICT-2014-1290; PICT-2016-1079). The Swiss NSF (P2NEP3_178543) to D.S. We would like to acknowledge also Sergio Pérez Ortega and Raquel Pino (Real Jardín Botánico de Madrid, CSIC, Spain) for the fruitful discussions on trebouxiophyceae and symbioses in lichens, and two anonymous reviewers for valuable comments on the manuscript.

\section{References}

Aboal, M., and Werner, O. (2011) Morphology, fine structure, life cycle and phylogenetic analysis of Phyllosiphon arisari, a siphonous parasitic green alga. Eur J Phycol 46: 181-192.

Altschul, S., Madden, T.L., Schäffer, A.A., Zhang, J., Zhang, Z., Miller, W., and Lipman, D.J. (1997) Gapped BLAST and PSIBLAST: a new generation of protein database search programs. Nucleic Acids Res 25: 3389-3402.

Amaral-Zettler, L.A., McCliment, E.A., Ducklow, H.W., and Huse, S.M. (2009) A method for studying protistan diversity using massively parallel sequencing of V9 hypervariable regions of small-subunit ribosomal RNA genes. PLOS ONE: 4: e6372.

Banerjee, A., Sharma, R., Chisti, Y., and Banerjee, U.C. (2002) Botryococcus braunii: a renewable source of hydrocarbons and other chemicals. Crit Rev Biotechnol 22: 245-279.

Berger, S.A., and Stamatakis, A. (2011) Aligning short reads to reference alignments and trees. Bioinformatics 27: 2068-2075.

Blanc, G., Agarkova, I., Grimwood, J., Kuo, A., Brueggeman, A., Dunigan, D.D., et al. (2012) The genome of the polar eukaryotic microalga Coccomyxa subellipsoidea reveals traits of cold adaptation. Genome Biol 13: R39.

Bock, C., Krienitz, L., and Pröschold, T. (2011) Taxonomic reassessment of the genus Chlorella (Trebouxiophyceae) using molecular signatures (barcodes), including description of seven new species. Fottea 11: 293-312.

Büdel, B., Darienko, T., Deutschewitz, K., Dojani, S., Friedl, T., Mohr, K.I., et al. (2009) Southern African biological soil crusts are ubiquitous and highly diverse in drylands, being restricted by rainfall frequency. Microb Ecol 57: 229-247.

Capella-Gutierrez, S., Silla-Martinez, J.M., and Gabaldon, T. (2009) trimAl: a tool for automated alignment trimming in large-scale phylogenetic analyses. Bioinformatics 25: 1972-1973.

Cavacini, P. (2001) Soil algae from northern Victoria Land (Antarctica). Polar Biosci 14: 45-60.

de Koning, A.P., and Keeling, P.J. (2006) The complete plastid genome sequence of the parasitic green alga Helicosporidium sp. is highly reduced and structured. BMC Biol 4: 12.

de Vargas, C., Audic, S., Henry, N., Decelle, J., Mahé, F., Logares, R., et al. (2015) Ocean plankton. Eukaryotic plankton diversity in the sunlit ocean. Science 348: 1261605.

de Vries, J., and Archibald, J.M. (2018) Plant evolution: landmarks on the path to terrestrial life. New Phytol 217: 1428-1434.

del Campo, J., Kolisko, M., Boscaro, V., Santoferrara, L.F., Nenarokov, S., Massana, R., et al. (2018) EukRef: phylogenetic curation of ribosomal RNA to enhance understanding of eukaryotic diversity and distribution. PLoS Biol 16: e2005849.

Edgar, R.C. (2010) Search and clustering orders of magnitude faster than BLAST. Bioinformatics 26: 2460-2461.

Fermani, P., Mataloni, G., and Van de Vijver, B. (2007) Soil microalgal communities on an antarctic active volcano (Deception Island, south Shetlands). Polar Biol 30: 1381-1393.

Flechtner, V.R., Pietrasiak, N., and Lewis, L.A. (2013) Newly revealed diversity of green microalgae from wilderness areas of Joshua tree national park (JTNP). Monogr West North Am Nat 6: 43-63.

Foflonker, F., Mollegard, D., Ong, M., Yoon, H.S., and Bhattacharya, D. (2018) Genomic analysis of Picochlorum species reveals how microalgae may adapt to variable environments. Mol Biol Evol 35: 2702-2711.

Fučíková, K., Lewis, P.O., and Lewis, L.A. (2014) Widespread desert affiliation of trebouxiophycean algae (Trebouxiophyceae, Chlorophyta) including discovery of three new desert genera. Phycol Res 62: 294-305.

Fuhrman, J.A., Steele, J.A., Hewson, I., Schwalbach, M.S., Brown, M.V., Green, J.L., and Brown, J.H. (2008) A latitudinal diversity gradient in planktonic marine bacteria. Proc Natl Acad Sci U S A 105: 7774-7778. 
Gomaa, F., Kosakyan, A., Heger, T.J., Corsaro, D., Mitchell, E.A.D., and Lara, E. (2014) One alga to rule them all: unrelated mixotrophic testate amoebae (Amoebozoa, Rhizaria and Stramenopiles) share the same symbiont (Trebouxiophyceae). Protist 165: 161-176.

Guillou, L., Bachar, D., Audic, S., Bass, D., Berney, C., Bittner, L., et al. (2013) The Protist ribosomal reference database (PR2): a catalog of unicellular eukaryote small sub-unit rRNA sequences with curated taxonomy. Nucleic Acids Res 41: D597-D604.

Guiry, M.D., and Guiry, G.M. (2018) AlgaeBase. National University of Ireland, Galway: World-wide electronic publication. http://www.algaebase.org.

Hawksworth, D. (2000) Freshwater and marine lichenforming fungi. Fungal Divers 5: 1-7.

Heesch, S., Sutherland, J.E., and Nelson, W.A. (2012) Marine Prasiolales (Trebouxiophyceae, Chlorophyta) from New Zealand and the Balleny Islands, with descriptions of Prasiola novaezelandiae sp. nov. and Rosenvingiella australis sp. nov. Phycologia 51: 217-227.

Hinojosa-Vidal, E., Marco, F., Martínez-Alberola, F., Escaray, F.J., García-Breijo, F.J., Reig-Armiñana, J., et al. (2018) Characterization of the responses to saline stress in the symbiotic green microalga Trebouxia sp. TR9. Planta 248: 1473-1486.

Hodač, L., Hallmann, C., Spitzer, K., Elster, J., Faßhauer, F., Brinkmann, N., et al. (2016) Widespread green algae Chlorella and Stichococcus exhibit polar-temperate and tropical-temperate biogeography. FEMS Microbiol Ecol 92: $1-16$.

Honegger, R., and Brunner, U. (1981) Sporopollenin in the cell walls of Coccomyxa and Myrmecia phycobionts of various lichens: an ultrastructural and chemical investigation. Can J Bot 59: 2713-2734.

Huss, V.A.R., Frank, C., Hartmann, E.C., Hirmer, M., Kloboucek, A., Seidel, B.M., et al. (1999) Biochemical taxonomy and molecular phylogeny of the genus Chlorella sensu lato (Chlorophyta). J Phycol 35: 587-598.

Juárez, Á.B., Vélez, C.G., Iñiguez, A.R., Martínez, D.E., Rodríguez, M.C., Vigna, M.S., and de Molina, M.d.C.R. (2011) A Parachlorella kessleri (Trebouxiophyceae, Chlorophyta) strain from an extremely acidic geothermal pond in Argentina. Phycologia 50: 413-421.

Katoh, K., and Standley, D.M. (2013) MAFFT multiple sequence alignment software version 7: improvements in performance and usability. Mol Biol Evol 30: 772-780.

Krienitz, L., Huss, V.A.R., and Bock, C. (2015) Chlorella: 125 years of the green survivalist. Trends Plant Sci 20: 67-69.

Lara, E., and Gomaa, F. (2017) Symbiosis between testate amoebae and photosynthetic organisms. In Algal and Cyanobacteria Symbioses. New Jersey, USA: World Scientific (Europe), pp. 399-419.

Lass-Flörl, C., and Mayr, A. (2007) Human protothecosis. Clin Microbiol Rev 20: 230-242.

Lee, J.J., Reidy, J., and Kessler, E. (1982) Symbiotic chlorella species from larger foraminifera. Bot Mar 25: 171-176.

Legendre, P., and Gallagher, E.D. (2001) Ecologically meaningful transformations for ordination of species data. Oecologia 129: 271-280.

Leliaert, F., Smith, D.R., Moreau, H., Herron, M.D., Verbruggen, H., Delwiche, C.F., and De Clerck, O. (2012)
Phylogeny and molecular evolution of the Green algae. CRC Crit Rev Plant Sci 31: 1-46.

Letunic, I., and Bork, P. (2007) Interactive tree of life (iTOL): an online tool for phylogenetic tree display and annotation. Bioinformatics 23: 127-128.

Lewis, L.A., and McCourt, R.M. (2004) Green algae and the origin of land plants. Am J Bot 91: 1535-1556.

Lemieux, C., Otis, C., and Turmel, M. (2014) Chloroplast phylogenomic analysis resolves deep-level relationships within the green algal class Trebouxiophyceae. BMC Evol Biol 14: 211.

Logares, R., Bråte, J., Bertilsson, S., Clasen, J.L., ShalchianTabrizi, K., and Rengefors, K. (2009) Infrequent marinefreshwater transitions in the microbial world. Trends Microbiol 17: 414-422.

Logares R. ramalok/amplicon_processing: Workflow for Analysing MiSeq Amplicons based on Uparse. 2017, doi: https://doi.org/10.5281/ZENODO.259579.

Letsch, M.R., Muller-Parker, G., Friedl, T., and Lewis, L.A. (2009) Elliptochloris marina sp. nov.(Trebouxiophyceae, Chlorophyta), symbiotic green alga of the temperate pacific sea anemones Anthopleura xanthogrammica and A. elegantissima (Anthozoa, Cnidaria) 1. J Phycol 45: 1127-1135.

Mahé, F., Rognes, T., Quince, C., de Vargas, C., and Dunthorn, M. (2014) Swarm: robust and fast clustering method for amplicon-based studies. PeerJ 2: e593.

Mahé, F., de Vargas, C., Bass, D., Czech, L., Stamatakis, A., Lara, E., et al. (2017) Parasites dominate hyperdiverse soil protist communities in Neotropical rainforests. Nat Ecol Evol 1: 1-8.

Metz, S., Lopes dos Santos, A., Berman, M.C., Bigeard, E., Licursi, M., Not, F., et al. (2019) Diversity of photosynthetic picoeukaryotes in eutrophic shallow lakes as assessed by combining flow cytometry cell-sorting and high throughput sequencing. FEMS Microbiol Ecol 95: fiz038.

Metzger, P., Allard, B., Casadevall, E., Berkaloff, C., and Coute, A. (1990) Structure and chemistry of a new chemical race of Botryococcus braunii (chlorophyceae) that produces lycopadiene, a tetraterpenoid hydrocarbon. J Phycol 26: 258-266.

Metzger, P., and Largeau, C. (1999) Chemicals of Botryococcus braunii. In: Chemicals from microalgae. Cohen, Z. (ed.). London: Taylor \& Francis, pp. 205-260.

Metzger, P., Largeau, C., and Casadevall, E. (1991) Lipids and macromolecular lipids of the hydrocarbon-rich microalga Botryococcus braunii. Chemical structure and biosynthesis. In Geochemical and Biotechnological Importance. Vienna: Springer, pp. 1-70.

Neofotis, P., Huang, A., Sury, K., Chang, W., Joseph, F., Gabr, A., et al. (2016) Characterization and classification of highly productive microalgae strains discovered for biofuel and bioproduct generation. Algal Res 15: 164-178.

Neustupa, J., Němcová, Y., Veselá, J., Steinová, J., and Škaloud, P. (2013) Leptochlorella corticola gen. et sp. nov. and Kalinella apyrenoidosa sp. nov.: two novel Chlorella-like green microalgae (Trebouxiophyceae, Chlorophyta) from subaerial habitats. Int J Syst Evol Microbio/ 63: 377-387.

Oksanen J, Kindt R, Legendre P et al. The vegan package: community ecology package, version 1.13-1. 2008. http:// vegan.r-forge.r-project.org. 
Pan, J., del Campo, J., and Keeling, P.J. (2017) Reference tree and environmental sequence diversity of Labyrinthulomycetes. J Eukaryot Microbiol 64: 88-96.

Pálffy, K., Felföldi, T., Mentes, A., Horváth, H., Márialigeti, K., Boros, E., et al. (2014) Unique picoeukaryotic algal community under multiple environmental stress conditions in a shallow, alkaline pan. Extremophiles 18: 111-119.

Pombert, J.-F., and Keeling, P.J. (2010) The mitochondrial genome of the entomoparasitic green alga Helicosporidium. PLOS ONE 5: e8954.

Pröschold, T., Darienko, T., Silva, P.C., Reisser, W., and Krienitz, L. (2011) The systematics of Zoochlorella revisited employing an integrative approach. Environ Microbiol 13: 350-364.

Quast, C., Pruesse, E., Yilmaz, P., Gerken, J., Schweer, T., Yarza, P., et al. (2012) The SILVA ribosomal RNA gene database project: improved data processing and webbased tools. Nucleic Acids Res 41: D590-D596.

Raja, R., Hemaiswarya, S., Kumar, N.A., Sridhar, S., and Rengasamy, R. (2008) A perspective on the biotechnological potential of microalgae. Crit Rev Microbiol 34: 77-88.

Rambold, G., Friedl, T., and Beck, A. (1998) Photobionts in lichens: possible indicators of phylogenetic relationships? Bryologist 101: 392-397.

Schiaffino, M.R., Lara, E., Fernández, L.D., Balagué, V., Singer, D., Seppey, C.V.W., et al. (2016) Microbial eukaryote communities exhibit robust biogeographical patterns along a gradient of Patagonian and Antarctic lakes. Environ Microbiol 18: 5249-5264.

Safi, C., Zebib, B., Merah, O., Pontalier, P.-Y., and VacaGarcia, C. (2014) Morphology, composition, production, processing and applications of Chlorella vulgaris: a review. Renew Sust Energ Rev 35: 265-278.

Singer, D., Lara, E., Steciow, M.M., Seppey, C.V.W., Paredes, N., Pillonel, A., et al. (2016) High-throughput sequencing reveals diverse oomycete communities in oligotrophic peat bog micro-habitat. Fungal Ecol 23: 42-47.

Škaloud, P., Friedl, T., Hallmann, C., Beck, A., and Dal Grande, F. (2016) Taxonomic revision and species delimitation of coccoid green algae currently assigned to the genus Dictyochloropsis (Trebouxiophyceae, Chlorophyta). J Phycol 52: 599-617.

Seppey, C.V.W., Singer, D., Dumack, K., Fournier, B., Belbahri, L., Mitchell, E.A.D., and Lara, E. (2017) Distribution patterns of soil microbial eukaryotes suggests widespread algivory by phagotrophic protists as an alternative pathway for nutrient cycling. Soil Biol Biochem 112: 68-76. https://doi. org/10.1016/j.soilbio.2017.05.002.
Stamatakis, A. (2014) RAxML version 8: a tool for phylogenetic analysis and post-analysis of large phylogenies. Bioinformatics 30: 1312-1313.

Tragin, M., Zingone, A., and Vaulot, D. (2018) Comparison of coastal phytoplankton composition estimated from the V4 and V9 regions of the 18S rRNA gene with a focus on photosynthetic groups and especially Chlorophyta. Environ Microbiol 20: 506-520.

Tragin, M., and Vaulot, D. (2018) Green microalgae in marine coastal waters: the ocean sampling day (OSD) dataset. Sci Rep 8: 14020.

Thüs, H., Muggia, L., Pérez-Ortega, S., Favero-Longo, S.E., Joneson, S., O'Brien, H., et al. (2011) Revisiting photobiont diversity in the lichen family Verrucariaceae (Ascomycota). Eur J Phycol 46: 399-415.

Trémouillaux-Guiller, J., and Huss, V.A.R. (2007) A cryptic intracellular green alga in Ginkgo biloba: ribosomal DNA markers reveal worldwide distribution. Planta 226: 553-557.

Zhang, J., Kapli, P., Pavlidis, P., and Stamatakis, A. (2013) A general species delimitation method with applications to phylogenetic placements. Bioinformatics 29: 2869-2876.

\section{Supporting Information}

Additional Supporting Information may be found in the online version of this article at the publisher's web-site:

Fig. S1. Rarefaction curve for each environment and for all sequences together respectively.

Table S1. Sequences retrived from public databases with all their information obteined by genbank and culture collections databases.

Table S2. Final samples analysed in this study. For marine samples reference to de Vargas et al., 2015.

Table S3. Taxonomy assignation for each OTU using $\mathrm{PR}^{2}$ as reference database. The reference corresponded to NCBI accession Number for new OTUs from this work (MK988625 to MK989495) and the reference paper where the OTUs were extracted.

Table S4. Trebouxiophyceae OTUs contribution to each sample. Table S5 Representatives OTUs for each environment. Clade and OTUs that contribute with more than $1 \%$ at least one environment or in the total reads for each clade were showed. The number is the percentage of contribution for each OTUs to each environment. 